\title{
Indicadores sanguíneos de daño hepático en novillos cruza cebú parasitados por Fasciola hepatica
}

\author{
Mussart, N.B.; Coppo, J.A. \\ Cátedra de Fisiología, Facultad de Ciencias Veterinarias, UNNE, Sargento Cabral 2139, \\ Corrientes (3400), Argentina. Tel/fax: 03783-425753. E-mail: jcoppo@vet.unne.edu.ar.
}

\begin{abstract}
Resumen
Mussart, N.B.; Coppo, J.A.: Indicadores sanguíneos de daño hepático en novillos cruza cebú parasitados por Fasciola hepatica. Rev. vet. 20: 2, 81-85, 2009. El ensayo tuvo como objetivo indagar eventuales cambios de los indicadores sanguíneos de daño hepático durante la fase subclínica (asintomática) de la fasciolosis. Se seleccionaron 56 muestras de sangre de novillos cruza cebú aparentemente sanos, en mataderos de zonas enzoóticas de la Provincia de Corrientes (Argentina). Posteriormente al sacrificio se estableció que 29 de ellos albergaba escasa a moderada cantidad de duelas en el hígado, permaneciendo libres del parásito los restantes. Con técnicas convencionales de laboratorio se efectuó eritrograma, leucograma, proteinograma, enzimograma (fosfatasa alcalina ALP, gammaglutamil transferasa GGT, aspartato aminotransferasa AST) y determinaciones de hierro, glucosa, bilirrubina y tiempo de protrombina. Los animales parasitados revelaron valores más elevados de leucocitos, eosinófilos, gamma globulinas y GGT que los controles $(p<0,05)$, signos inflamatorios que se imputan a una incipiente colangitis, propia de la fase subclínica de la distomatosis. No se verificaron cambios atribuibles a colestasis ni insuficiencia hepática.
\end{abstract}

Palabras clave: novillo, fasciolosis, daño hepático, hematología, bioquímica.

\begin{abstract}
Mussart, N.B.; Coppo, J.A.: Liver damage hematological indicators in half-bred Zebu steers parasitized by Fasciola hepatica. Rev. vet. 20: 2, 81-85, 2009. The objective of the trial was to investigate eventual changes of hepatic damage blood indicators, during the subclinical (asymptomatic) period of fasciolosis. Fifty six blood samples of apparently healthy half-bred Zebu steers were collected in slaughterhouses from enzootic areas of Corrientes Province (Argentina). After slaughtering it was verified that 29 of them harbored scarce to moderate amount of liver flukes, being the rest of the animals free of parasites. Erythrogram, leukogram, proteinogram, enzymogram (alkaline phosphatase ALP, gammaglutamyl transferase GGT, aspartate aminotransferase AST) and iron, glucose, bilirubin and prothrombin time were determined by conventional laboratory techniques. Leukocyte, eosinophil, gamma globulin, and GGT values were higher in parasitized animals than in controls $(p<0.05)$. Such inflammatory signs are imputed to an incipient cholangitis, which is characteristic of the subclinical period of liver fluke disease. Changes attributable to cholestasis and hepatic failure were not verified.
\end{abstract}

Key words: steer, fasciolosis, liver damage, hematology, biochemistry.

\section{INTRODUCCIÓN}

La distomatosis o fasciolosis es una enfermedad parasitaria originariamente hepática que puede llegar a causar la muerte del huésped por complicaciones graves. Su agente etiológico es el trematode Fasciola hepatica (distoma, duela del hígado, liver fluke, saguaypé), el cual parasita a todos los mamíferos, incluyendo al hombre $3,8,11,14$.

La OMS considera que actualmente existen dos millones y medio de personas infestadas ${ }^{14}$. La pobla-

Recibido: 15 junio 2009 / Aceptado: 29 julio 2009 ción de Sudamérica se encuentra en riesgo debido a sus hábitos alimentarios y deficientes condiciones de saneamiento ${ }^{11}$. El primer caso de infestación humana en Argentina fue detectado en Resistencia (Chaco) en la década de $1950{ }^{13}$. Luego se registraron varios casos más, entre ellos los de las provincias de San Luis ${ }^{2}$ y Neuquén ${ }^{15}$. Estas comunicaciones atestiguan que bioquímicos y médicos fueron pioneros en la tarea de establecer los cambios sanguíneos provocados por la parasitosis.

En medicina veterinaria la distomatosis afecta principalmente a los rumiantes, causando ingentes pérdidas de la producción animal ${ }^{3}$. En determinadas áreas de 
Argentina la prevalencia es del $82 \%$ en ovinos, $86 \%$ en bovinos y $100 \%$ en caprinos ${ }^{15}$. En la Provincia de Corrientes la prevalencia de fasciolosis en rumiantes aumentó significativamente durante las dos últimas décadas; en la actualidad el área afectada abarca principalmente una ancha franja aplicada sobre la orilla del río Paraná ${ }^{12}$, incluyendo varios departamentos de donde provinieron las muestras utilizadas en este estudio. En esta zona se crían primordialmente bovinos cruza cebú (Bos indicus), a quienes se les atribuye el hecho de ser más resistentes a los parásitos que el ganado británico (Bos taurus) ${ }^{16}$.

En el nordeste argentino el ganado infestado por distomatosis revela los primeros síntomas al finalizar el otoño, cuando los fasciolómulos invaden el parénquima hepático (cólico, fiebre). La hiperplasia de la mucosa canalicular y los propios parásitos generan obstrucción de conductos biliares e ictericia (Figura 1). Los hábitos hematófagos y las sustancias tóxicas liberadas por el trematode destruyen eritrocitos circulantes, en tanto que la migración del parásito a través del parénquima y la hipertensión canalicular conducen a la inflamación y necrosis de tejido hepático, con consiguiente insuficiencia funcional. Al agotarse el glucógeno sobreviene la adinamia, que obliga a obtener energía a partir de proteínas y lípidos. Al extinguirse tales combustibles hará su aparición la caquexia típica de la enfermedad. La hipoproteinemia originará edemas y ascitis. Finalmente las áreas inflamatorio-necróticas del hígado sufren fibrosis y atrofia, pudiendo evolucionar hacia la cirrosis hepática ${ }^{3}$.

Tales lesiones deberían alterar indicadores sanguíneos capaces de evidenciar anemia (eritrocitos, hemoglobina, hematocrito, hierro, índices hematimétricos), colestasis (bilirrubina total y directa, actividades de fosfatasa alcalina ALP y gammaglutamil transferasa GGT), inflamación (leucocitosis, aspartato aminotransferasa AST, ex-GOT), aparición de antiparasitarios naturales (eosinofilia), elevación de anticuerpos (gamma globulinas) e instalación de insuficiencia hepática (protrombina, glucosa, albúminas y proteínas totales) ${ }^{5}$.

En rumiantes se preconiza asimismo el uso diagnóstico de la actividad glutamato dehidrogenasa GLDH, cuyo aumento indicaría daño severo de los hepatocitos ${ }^{4}$, 7,8, 17 . En la fasciolosis del hombre se elevarían las actividades alanin aminotransferasa ALT (ex-GPT) y sorbitol dehidrogenasa $\mathrm{SDH}^{8}$. La arginasa ARG fue hallada con valores altos en vacas ${ }^{6}$ pero con valores normales en terneros ${ }^{18}$. No serían afectados los valores de amilasa, creatinina ni electrolitos séricos ${ }^{10}$. Se asevera que para el diagnóstico de distomatosis las enzimas séricas son más precoces que el hallazgo de huevos en las heces ${ }^{18}$.

La fasciolosis se caracterizaría por evolucionar desde una fase aguda, que comenzaría a las dos semanas nica de la enfermedad, en novillos asintomáticos cuyo mestizaje con ganado índico vaticina mayor grado de resistencia a la parasitosis.

\section{MATERIAL Y MÉTODOS}

Se colectaron muestras de sangre de 60 novillos media sangre cebú x británico, cuyo examen clínico no reveló síntomas de enfermedad. Su estado nutricional era aceptable, variando el peso vivo entre 350 a 450 $\mathrm{kg}$. La venopunción yugular se efectuó previamente al sacrificio de los animales, en mataderos y frigoríficos de los departamentos de San Roque, Concepción, Bella Vista y Goya (Provincia de Corrientes, Argentina). A posteriori del examen macrocópico del hígado, se seleccionaron 30 muestras negativas y 30 positivas a $F$. hepatica. En cuatro casos las muestras debieron descartarse por hemólisis.

En sangre total tratada con EDTA se efectuaron eritrogramas y leucogramas. Hematocrito, concentraciones de hemoglobina, eritrocitos y leucocitos, así como determinación de los índices hematimétricos (volumen corpuscular medio VCM, hemoglobina corpuscular media HCM y concentración de hemoglobina corpuscular media CHCM), se realizaron en un contador hematológico electrónico. La fórmula leucocitaria se obtuvo a partir de frotis coloreados con Giemsa y el hierro sérico por fotocolorimetría (técnica de la ferrozina, reactivos Wiener Lab).

A partir del suero, mediante espectrofotometría se determinaron las actividades ALP (fenilfosfato), GGT (nitroanilida) y AST (aspartato-oxoglutarato), así como las concentraciones de proteínas totales (biuret), glucosa (oxidasa-peroxidasa) y bilirrubina total y directa (diazorreactivo sulfanílico). Las tasas de albúminas y globulinas alfa, beta y gamma se obtuvieron por electroforesis y ulterior densitometría; la relación albúminas / globulinas (RAG) se obtuvo por cálculo. El tiempo de protrombina se determinó según técnica de Quick. En todos los casos se utilizaron reactivos Wiener Lab.

Las estadísticas se realizaron en el marco de un diseño completamene aleatorizado. La distribución normal fue verificada por test de Wilk-Shapiro. Constata- 
da la simetría gaussiana, se aplicaron estadísticas descriptivas paramétricas de tendencia central (media aritmética) y dispersión (desvío estándar). El análisis de la variancia (ANOVA) para cada variable dependiente se efectuó por modelo lineal a un criterio, previa verificación de homogeneidad de la variancia (test de Bartlett). Los cálculos se realizaron con el auxilio de un programa informático (Statistix 1996). Para todas las inferencias se estipuló un nivel de riesgo alfa del $5 \%$, por debajo del cual se rechazó la hipótesis nula de igualdad.

\section{RESULTADOS}

Del total de muestras aptas para los análisis de laboratorio, 29 de ellas correspondieron a novillos que albergaban ejemplares juveniles y adultos de $F$. hepatica en el tejido hepático, en tanto que los 27 restantes fueron negativos. Ningún animal reveló enfermedades intercurrentes capaces de alterar los indicadores sanguíneos de daño hepático. Los casos positivos asumieron categoría de leves, por la escasa cantidad de parásitos hallada y las exiguas lesiones anatómicas constatadas.

En cada variable dependiente la distribución de los datos $(\mathrm{n}=56)$ fue normal, lo cual facultó el uso de estadísticas paramétricas; por su parte el test de Bartlett reveló que las variancias fueron homogéneas, habilitando la aplicación del ANOVA.

La Tabla 1 exhibe los valores sanguíneos de las series roja y blanca. El análisis de los parámetros eritrocitarios muestra que no existieron diferencias significativas entre animales positivos y negativos, no obstante lo cual se advierte que todos los ejemplares parasitados revelaron valores más bajos que los controles. Inversamente, leucocitos totales y eosinófilos fueron significativamente más elevados en animales parasitados que en controles, sin grandes diferencias en el resto del recuento diferencial. Los neutrófilos no revelaron desviación izquierda.

Los parámetros indicadores de obstrucción biliar se muestran en la Tabla 2. La actividad sérica ALP, así como las fracciones directa, indirecta y total de bilirrubina no revelaron diferencias significativas entre grupos, aunque en todos los casos los valores fueron más altos en novillos positivos a $F$. hepatica. En cambio, la enzima GGT asumió valores significativamente más elevados en el grupo de animales parasitados, excediendo el límite superior del intervalo de referencia.

En la Tabla 3 se incluyen los marcadores de lesiones inflamatorio-necróticas del hígado, destacándose el significativo aumento de las gamma globulinas en el lote parasitado. Al contrario, alfa y beta globulinas,
Tabla 1. Valores hemáticos de las series roja y blanca obtenidos en animales positivos y negativos a $F$. hepatica.

\begin{tabular}{lcccc}
\hline parámetro & interv. ref. ${ }^{(5)}$ & $-(\mathrm{n}=27)$ & $+(\mathrm{n}=29)$ & signif. \\
\hline hematocrito (\%) & $40-45$ & $43,2 \pm 2,6$ & $41,7 \pm 3,5$ & - \\
hemoglobina (g/dl) & $13-15$ & $13,6 \pm 1,4$ & $12,9 \pm 1,1$ & - \\
eritrocitos (T/l) & $7,8-8,8$ & $7,97 \pm 0,6$ & $7,88 \pm 0,7$ & - \\
VCM (fl) & $45-55$ & $54 \pm 4$ & $52 \pm 6$ & - \\
HCM (pg) & $15-20$ & $17 \pm 3$ & $16 \pm 2$ & - \\
CHCM (\%) & $26-33$ & $32 \pm 2$ & $31 \pm 2$ & - \\
hierro (ug/dl) & $106-127$ & $120 \pm 12$ & $116 \pm 14$ & - \\
leucocitos (G/l) & $9-10$ & $9,22 \pm 1,7$ & $12,6 \pm 2,2$ & 0,05 \\
neutrófilos (\%) & $26-30$ & $30,9 \pm 2,8$ & $28,4 \pm 2,5$ & - \\
linfocitos (\%) & $57-65$ & $59,2 \pm 4,1$ & $55,2 \pm 3,8$ & - \\
monocitos (\%) & $4-6$ & $4,8 \pm 0,3$ & $4,0 \pm 0,5$ & - \\
eosinófilos (\%) & $5-7$ & $5,1 \pm 0,4$ & $12,4 \pm 1,7$ & 0,02 \\
basófilos (\%) & $0-1$ & - & - & - \\
\hline
\end{tabular}

-: negativo, +: positivo, interv. ref.: intervalo de referencia, signif.: significación, VCM: volumen corpuscular medio, HCM: hemoglobina corpuscular media, CHCM: concentración de HCM, T/1: tera por litro, G/l: giga por litro.

Tabla 2. Valores séricos indicadores de colestasis en animales positivos y negativos a $F$. hepatica.

\begin{tabular}{lcccc}
\hline parámetro & interv. ref. $^{(5)}$ & $-(\mathrm{n}=27)$ & $+(\mathrm{n}=29)$ & signif. \\
\hline ALP (UI/l) & $205-248$ & $216 \pm 11$ & $224 \pm 17$ & - \\
GGT (UI/l) & $25-33$ & $27,4 \pm 2,8$ & $39,1 \pm 4,6$ & 0,02 \\
BRR indirecta (mg/l) & hasta 4 & $2,88 \pm 0,2$ & $3,10 \pm 0,3$ & - \\
BRR directa (mg/l) & hasta 1 & $0,76 \pm 0,03$ & $0,97 \pm 0,04$ & - \\
BRR total (mg/l) & hasta 5 & $3,64 \pm 0,4$ & $4,07 \pm 0,3$ & - \\
\hline
\end{tabular}

-: negativo, +: positivo, interv. ref.: intervalo de referencia, signif.: significación, ALP: fosfatasa alcalina, GGT: gammaglutamil transferasa, BRR: bilirrubina, * también puede elevarse en procesos inflamatorios del hígado ${ }^{5}$.

Tabla 3. Valores séricos indicadores de inflamación y necrosis en animales positivos y negativos a $F$. hepatica.

\begin{tabular}{lcccc}
\hline parámetro & interv. ref. ${ }^{(5)}$ & $-(\mathrm{n}=27)$ & $+(\mathrm{n}=29)$ & signif. \\
\hline AST $(\mathrm{UI} / \mathrm{l})$ & $39-57$ & $41,7 \pm 3,6$ & $44,1 \pm 3,9$ & - \\
alfa glob. $(\mathrm{g} / \mathrm{dl})$ & $0,6-0,7$ & $0,64 \pm 0,08$ & $0,61 \pm 0,06$ & - \\
beta glob. $(\mathrm{g} / \mathrm{dl})$ & $0,8-0,9$ & $0,85 \pm 0,13$ & $0,83 \pm 0,11$ & - \\
gamma glob. $(\mathrm{g} / \mathrm{dl})$ & $2,3-2,6$ & $2,41 \pm 0,36$ & $3,60 \pm 0,41$ & 0,03 \\
\hline
\end{tabular}

-: negativo, +: positivo, interv. ref.: intervalo de referencia, signif.: significación, AST: aspartato aminotransferasa, glob.: globulinas, RAG: relación albúminas/globulinas.

así como la actividad sérica AST, permanecieron encuadradas en sus intervalos de referencia, aunque las dos últimas fueron ligeramente más altas en animales positivos a $F$. hepatica.

En la Tabla 4 se consignan los indicadores de insuficiencia hepática. El tiempo de protrombina y las concentraciones séricas de glucosa y albúminas no registraron diferencias significativas entre lotes. En cambio, en el grupo parasitado las proteínas totales fueron más altas y la RAG más baja, abandonando los límites de sus respectivos intervalos de referencia. 
Tabla 4. Valores séricos indicadores de insuficiencia hepática en animales positivos y negativos a $F$. hepatica.

\begin{tabular}{lcccc}
\hline parámetro & interv. ref. $^{(5)}$ & $-(\mathrm{n}=27)$ & $+(\mathrm{n}=29)$ & signif. \\
\hline t. de protrombina (seg) & $10-25$ & $16,4 \pm 1,8$ & $19,2 \pm 1,5$ & - \\
glucosa $(\mathrm{g} / \mathrm{l})$ & $0,58-0,71$ & $0,61 \pm 0,07$ & $0,68 \pm 0,08$ & - \\
albúminas $(\mathrm{g} / \mathrm{dl})$ & $3,3-3,8$ & $3,72 \pm 0,38$ & $3,31 \pm 0,35$ & - \\
proteínas totales $(\mathrm{g} / \mathrm{dl})$ & $7,0-8,0$ & $7,62 \pm 0,81$ & $8,35 \pm 0,90$ & 0,05 \\
RAG (score) & $0,7-0,9$ & $0,95 \pm 0,09$ & $0,66 \pm 0,07$ & 0,05 \\
\hline
\end{tabular}

-: negativo, +: positivo, interv. ref.: intervalo de referencia, signif.: significación, t.: tiempo, RAG: relación albúminas / globulinas.

\section{DISCUSIÓN}

Los parámetros de la serie roja no se apartaron del intervalo de referencia estipulado para la categoría de ganado y zona geográfica. Si bien los valores de hematocrito, eritrocitos, hemoglobina, hierro e índices hematimétricos fueron más bajos en los ejemplares parasitados, la magnitud de su descenso no llegó a configurar el estado de anemia reportado para bovinos con fasciolosis $^{1,8}$, especialmente hipocrómica ${ }^{5,9}$. Para la distomatosis del ser humano se han citado reducciones tan bajas como $31 \%$ de hematocrito y $10 \mathrm{~g} / \mathrm{dl}$ de hemoglobina ${ }^{10}$.

En vacunos con fasciolosis versus controles sanos se registraron significativos descensos de hierro, hematocrito, hemoglobina, HCM y CHCM, con VCM normal, tipificando una anemia hipocrómica microcítica ${ }^{9}$ . Inversamente, en otros trabajos las disminuciones de hierro, hematocrito y eritrocitos se acompañaron del aumento de VCM, por lo cual la anemia fue macrocítica ${ }^{6}$.

Los datos aquí obtenidos para la serie blanca de animales parasitados con $F$. hepatica se acercan a los reportados en otras investigaciones, en las cuales el ganado afectado reveló leucocitosis y eosinofilia ${ }^{1,3}$. La media aritmética alcanzada en el presente trabajo por los eosinófilos de novillos parasitados (12,4\%) fue menor que el porcentaje comunicado por otro autor, del $17 \%{ }^{3}$.

En la infestación humana, eosinofilia y leucocitosis se elevan a niveles tan altos como $46 \%$ y $24.000 \mathrm{G} / 1$ respectivamente ${ }^{15}$, aunque en otros reportes se citan valores más bajos, de $4 \%$ y $11.300 \mathrm{G} / 1$ para sendos parámetros ${ }^{10}$. La elevación de los eosinófilos parece ser una constante de la distomatosis humana ${ }^{2}$. En ganado con fasciolosis la eosinofilia fue asociada con disminución de neutrófilos, linfocitos y monocitos ${ }^{6}$.

La ausencia de ictericia en los novillos parasitados estuvo acorde con la invariabilidad de la bilirrubinemia. La concentración sérica de bilirrubina se elevó en casos de obstrucción biliar severa ${ }^{1}$, especialmente la fracción directa ${ }^{5}$. En la distomatosis humana la bilirrubina total permaneció normal en algunos casos ${ }^{15}$ y en otros aumentó unas cuatro veces, a expensas de la fracción directa ${ }^{10}$. En el ganado con síntomas de fasciolosis, la hiperbilirrubinemia frecuentemente se acompaña de bilirrubinuria ${ }^{3}$. Algunos investigadores aseveran que el aumento del pigmento biliar no es un hallazgo frecuente en el suero de los rumiantes afec$\operatorname{tados}^{8}$.
Las elevaciones séricas de las actividades ALP y GGT indican principalmente estados de colestasis, aunque la última de las enzimas nombradas también puede aumentar por inflamación canalicular o parenquimatosa ${ }^{5}$. En seres humanos afectados por la duela del hígado, ALP fue capaz de incrementarse dos veces en algunos casos ${ }^{10} \mathrm{y}$ hasta seis veces en otros ${ }^{15}$ . En algunas investigaciones también se la halló elevada en el ganado ${ }^{1,9}$. Los aumentos de GGT en la distomatosis ocurren en el hombre y en los bovinos ${ }^{7-9,18}$, en los cuales operaría como indicador de la fase subaguda de la enfermedad, cuando los trematodes obstruyen e inflaman los canalículos biliares ${ }^{4}$. La actividad AST, marcadora de daño parenquimatoso en la distomatosis humana ${ }^{10,15}$, también fue hallada elevada en bovinos afectados por dicha parasitosis $^{7,9,18}$, especialmente en la fase aguda ${ }^{8}$.

El aumento de gamma globulinas indicaría la respuesta inmunológica del organismo ante la presencia de $F$. hepatica, incrementando la tasa de inmunoglobulinas circulantes ${ }^{5}$. Tal circunstancia fue anteriormente corroborada en bovinos, con la consiguiente disminución de $\mathrm{RAG}^{6}$, aunque tal hallazgo no fue registrado en personas, en las cuales la concentración de proteínas totales fue normal ${ }^{10}$. En el ganado afectado las proteínas totales disminuirían debido a su utilización con fines energéticos ${ }^{3}$. El significativo incremento de las proteínas totales registrada en el presente ensayo debe necesariamente atribuirse al antemencionado aumento de gamma globulinas, que a su vez fue el responsable de la disminución de la RAG.

Al no verificarse aumento del tiempo de protrombina ni disminuciones de albúminas ni glucosa, se torna improbable la existencia de insuficiencia hepática en los bovinos parasitados ${ }^{5}$. En rumiantes gravemente parasitados por $F$. hepatica se encontraron disminuciones séricas de glucosa ${ }^{3} \mathrm{y}$ albúminas ${ }^{6}$, principalmente en la fase crónica de la enfermedad ${ }^{8}$. En una comunicación de distomatosis humana, glucosa y albúminas permanecieron en valores normales pero el tiempo de protrombina excedió al doble de su nivel de referencia ${ }^{10}$.

Sintetizando los cambios aquí registrados en los indicadores bioquímicos de novillos asintomáticos cuyo hígado hospedaba ejemplares de $F$. hepatica, surge que estuvieron ausentes los síndromes de colestasis e insuficiencia hepática, en tanto que leucocitosis, eosinofilia, hipergammaglobulinemia y aumento de la actividad GGT señalaron solamente la existencia de un estado inflamatorio, que quizás coincida con la "fase subclínica" (colangitis incipiente) propuesta por un prestigioso parasitólogo para los animales recientemente infestados con escasa cantidad de parásitos, que aún permanecen aparentemente sanos ${ }^{8}$. La supuesta mayor resistencia a los parásitos ${ }^{16}$ no preservaría al ganado media sangre cebú de la infestación por $F$. hepatica.

En conclusión, los cambios de los indicadores sanguíneos de daño hepático por $F$. hepatica en la fase subclínica de la enfermedad señalan la existencia de un 
moderado proceso inflamatorio que se interpreta como colangitis y que no cursó con síntomas clínicamente detectables en los novillos estudiados.

\section{REFERENCIAS}

1. Abu-Zeid YA. 2006. Helmintos tisulares. http://www. scribd.com/doc/7219085/Seminario-4-2006.

2. Ale D, Merciai G, Gil Echeverría M. 2000. Fasciola hepatica. Intensificación de su búsqueda en pacientes con eosinofilia y epidemiología positiva. Anales III Congreso Argentino de Parasitología, Mar del Plata (Argentina), p. 421.

3. Boero JJ. 1967. Parasitosis animales, Eudeba, Buenos Aires, p. 360-362.

4. Cardozo Estrela H. 2003. Diagnóstico de Fasciola hepática. http://produccionbovina.com/sanidad_intoxicaciones_metabolicos/parasitarias/parasitarias_bovinos/44diagnostico_fasciola_hepatica.htm.

5. Coppo JA. 2001. Fisiología comparada del medio interno, Ed. Dunken, Buenos Aires, p. 191-199 y 289-290.

6. Haroun EM, Hussein MF. 1975. Clinico-pathological studies on naturally-occurring bovine fascioliasis in the Sudan. J Helminthol 49: 143-152.

7. Hutchinson GW, Dawson K, Fitzgibbon CC, Martin PJ. 2009. Efficacy of an injectable combination anthelmintic (nitroxynil+clorsulon+ivermectin) against early immature Fasciola hepatica compared to triclabendazole combination flukicides given orally or topically to cattle. Vet Parasitol 162: 278-284.

8. Kassai T. 2002. Helmintología veterinaria, Acribia, Zaragoza, p. 5-11.

9. Lotfollahzadeh S, Mohri M, Bahadori SR, Dezfouly MR, Tajik P. 2008. The relationship between normocytic hypochromic anaemia and iron concentration together with hepatic enzyme activities in cattle infected with Fasciola hepatica. J Helminthol 82: 85-88.
10. Maco V, Marcos L, Montenegro J, Bellido J, Terashima A, Gotuzzo E. 2003. Obstruction of kehr drain due to Fasciola hepatica in a post-cholecystectomized patient secondary to acute cholangitis. Parasitol Latinoam 58: 152-158.

11. Mas-Coma MS, Esteban JG, Bargues MD. 1999. Epidemiology of human fascioliasis: a review and proposed new classification. Bull World Health Organ 77: 340-346.

12. Moriena RA, Racioppi O, Alvarez JD, Lombardero OJ. 2002. Distomatosis hepática (Fasciola hepatica) y otros trematodos de los rumiantes. Distribución en la provincia de Corrientes. Anales de la Reunión de Comunicaciones Cientificas y Tecnológica de la UNNE (Corrientes, Argentina), p. V055.

13. Petraglia AA. 1954. Parasitosis humana por Fasciola hepatica. Primer caso que se describe en el noreste argentino. Act Trab Asoc Arg Enferm Transm 3: 47-48.

14. Rim HJ, Farag HF, Sommani S, Cross JH. 1994. Foodborne trematodes: ignored or emerging? Parasitol Today 10: 207-209.

15. Rubel D, Prepelitchi L, Kleiman F, Carnevale S, Wisnivesky-Colli C. 2005. Estudio del foco en un caso de fasciolosis humana en Neuquén. Medicina (Buenos Aires) 65: 207-212.

16. Sardar SA, Ehsan MA, Anower AK, Rahman MM, Islam MA. 2006. Incidence of liver flukes and gastro-intestinal parasites in cattle. Bangl J Vet Med 4: 39-42.

17. Urquart G, Armour J, Duncan J, Dunn A, Jennings F. 1999. Veterinary Parasitology, Ed. Blackwell Science, London, p. 103-112.

18. Wyckoff JH, Bradley RE. 1985. Diagnosis of Fasciola hepatica infection in beef calves by plasma enzyme analysis. Am J Vet Res 46: 1015-1019. 